\title{
Influence of surgical arch reconstruction methods on single ventricle workload in the Norwood procedure
}

\author{
Keiichi Itatani, MD, ${ }^{\text {a,b,c }}$ Kagami Miyaji, MD, PhD, ${ }^{\text {a }}$ Yi Qian, PhD, ${ }^{\text {,d }}$ Jin Long Liu, ME, ${ }^{\text {b }}$ \\ Tomoyuki Miyakoshi, ME, ${ }^{\mathrm{b}}$ Arata Murakami, MD, $\mathrm{PhD},{ }^{\mathrm{c}}$ Minoru Ono, MD, $\mathrm{PhD},{ }^{\mathrm{c}}$ and \\ Mitsuo Umezu, $\mathrm{PhD}^{\mathrm{b}}$
}

\begin{abstract}
Objective: The study objective was to evaluate various types of Norwood arch reconstruction methods and to show the factors that affect the cardiac workload of the single ventricle. The Norwood procedure is one of the most challenging congenital heart surgeries. Several aortic arch reconstruction techniques have been reported to avoid recoarctation, ensure coronary perfusion, and improve long-term outcomes. Inside the arch, complicated turbulent flow is generated; however, little is known about the cause of the disadvantageous inefficient flow and the surgical techniques to avoid it.
\end{abstract}

\begin{abstract}
Methods: We created patient-specific computational hemodynamic models of 9 patients who underwent different types of arch reconstruction methods. Four patients had aortic atresia, and 5 patients had aortic stenosis. Flow profiles were defined by echocardiography data corrected with body surface area. Turbulent pulsatile flow was analyzed with the finite volume method. Flow energy loss was calculated to estimate cardiac workload, and wall shear stress was calculated to estimate vessel wall stiffness increase.
\end{abstract}

Results: Recoarctation and acute arch angles increased wall shear stress and energy loss. In the patients with aortic atresia, a longitudinal incision toward the descending aorta was effective in creating a smooth arch angle. In the patients with aortic stenosis, arch repair with the Damus-Kaye-Stansel procedure in a single anastomotic site was effective in creating sufficient anastomosis space and a smooth arch angle.

Conclusions: Creation of a large anastomotic space and a smooth aortic arch angle reduced wall shear stress and energy loss, and should improve long-term cardiac performance after the Norwood procedure. (J Thorac Cardiovasc Surg 2012;144:130-8)

The Norwood procedure for hypoplastic left heart syndrome and related malformations is one of the most technically challenging and high-risk surgical procedures for patients with the congenital heart anomaly. ${ }^{1,2}$ The procedure's long-term outcomes, including interstage mortality, have not been satisfactory despite the recent progress in preoperative risk factor evaluations and intra- and postoperative patient management. ${ }^{3}$ Since the first description by Norwood and colleagues, ${ }^{4}$ several technical modifications have been introduced, including complete ductal excision, the use of prosthetic patch material (eg, a pulmonary homograft), ${ }^{5}$ reconstruction with no exogenous material,,${ }^{6,7}$ and the interdigitating technique. ${ }^{8}$ There have been reports on the influences of the techniques on the outcomes, including freedom from recoarctation, ${ }^{1,2,9,10}$ because recoarctation or

From the Department of the Cardiovascular Surgery, ${ }^{\text {a }}$ Kitasato University School of Medicine, Kanagawa, Japan; Center for Advanced Biomedical Science, ${ }^{\mathrm{b}}$ TWIns, Waseda University, Tokyo, Japan; Department of Cardiac Surgery, ${ }^{\mathrm{c}}$ Graduate School of Medicine, The University of Tokyo, Japan; and The Australian School of Advanced Medicine, Macquarie University, ${ }^{\mathrm{d}}$ Sydney, Australia.

Disclosures: Authors have nothing to disclose with regard to commercial support.

Received for publication March 24, 2011; accepted for publication Aug 4, 2011; available ahead of print Sept 12, 2011

Address for reprints: Kagami Miyaji, MD, PhD, 1-15-1 Kitasato, Sagamihara, Kanagawa 228-8555, Japan (E-mail: Kagami111@aol.com).

$0022-5223 / \$ 36.00$

Copyright (c) 2012 by The American Association for Thoracic Surgery doi:10.1016/j.jtcvs.2011.08.013 aortic arch obstruction after the Norwood procedure deteriorates the function of the single right ventricle, resulting in a high mortality rate. ${ }^{11}$ After the Norwood procedure, the reconstructed aortic arch increases wall stiffness and decreases distensibility, resulting in increased afterload and decreased cardiac ejection fraction. ${ }^{11,12}$ However, most of the previous reports mainly focused on avoiding the recoarctation or maintaining coronary perfusion, and little is known about the comprehensive mechanisms causing disadvantageous flow.

The reconstructed aortic arch develops complicated turbulent flow. ${ }^{13}$ Flow collision from the ascending aorta and main pulmonary artery occurs in patients with aortic stenosis (AS), whereas there is a reverse flow in the ascending aorta for the blood supply to the coronary arteries in patients with aortic atresia (AA). Because of the increased afterload caused by recoarctation or stiff vessel walls and the flow turbulence inside the aortic arch, the reconstructed arch itself causes an inefficient flow and consumes a large amount of energy, resulting in increased cardiac workload.

Patient-specific computational fluid dynamic (CFD) models have been used to demonstrate the hemodynamic details and energetic efficiency of patients with single ventricle physiology. ${ }^{14-22}$ Previous CFD studies were mainly applied to the Fontan circulation ${ }^{14-19}$ and aimed at evaluating energy loss (EL), which affects the cardiac workload. Other 


\author{
Abbreviations and Acronyms \\ $\mathrm{AA}=$ aortic atresia \\ AS $=$ aortic stenosis \\ BSA $=$ body surface area \\ $\mathrm{CFD}=$ computational fluid dynamic \\ $\mathrm{CT}=$ computed tomography \\ DKS = Damus-Kay-Stansel \\ $\mathrm{EL}=$ energy loss \\ TCPC $=$ total cavopulmonary connection \\ $\mathrm{TP}=$ total pressure \\ WSS $=$ wall shear stress
}

previous studies demonstrated the Norwood procedure with simple geometry models ${ }^{20,21}$ and discussed flow balance between the pulmonary and the systemic circulation in a parallel circulation. We have created patient-specific CFD models to calculate transient pulsatile flow with turbulence. ${ }^{13}$ The objective of the present study was to analyze flow details inside the reconstructed arch of various types of Norwood procedures, to evaluate the cardiac workload of the single systemic ventricle by calculating the factors affecting it (the EL causing cardiac workload and wall shear stress [WSS] causing decreased wall distensibility), and to measure the strategies to optimize the Norwood procedure.

\section{MATERIALS AND METHODS}

\section{Patients and Surgical Methods}

We analyzed 9 patients (4 with AA and 5 with AS) with different surgical procedures. Patients' ages and body size (body surface area [BSA]) are shown in Figure 1. The arch reconstruction procedure details are described as follows and in Figure 1. All patients were diagnosed with hypoplastic left heart syndrome or related anomaly with hypoplastic aortic arch.

\section{Patients With Aortic Atresia}

In patient 1 , after the ligation of the ductus arteriosus, a longitudinal incision in the lesser curvature side of the aortic arch was made from the ascending aorta to the descending aorta, across the isthmus. The aortic arch was reconstructed with a triangular-shaped pulmonary homograft (Figure 1, A). In patients 2 and 3, the isthmus and periductal tissue were completely excised. During the process, the left subclavian artery was ligated because its orifice was adjacent to the isthmus. A hemicircular endto-end anastomosis was made after a longitudinal incision was made from the lesser curvature site of the aortic arch to the ascending aorta. A triangular-shaped pulmonary homograft patch was used to reconstruct the aortic arch in patient 2 (Figure 1, B), and the right side of the main pulmonary artery was longitudinally incised and anastomosed to the aortic arch in patient 3 (Figure 1, C). In patient 4, the ascending aorta was transected and anastomosed in a side-to-side fashion to the main pulmonary artery creating an outflow trunk. The lesser curvature side of the aortic arch was incised to the descending aorta, with the removal of the coarctation ridge. An end-toside anastomosis of the aortic arch and the trunk created with the main pulmonary artery was made to reconstruct the arch (Figure 1,D).

\section{Patients With Aortic Stenosis}

In patient 5 , after the removal of the ductal tissue, the descending aorta was anastomosed to the distal ascending aorta in an end-to-side fashion, and a Damus-Kay-Stansel (DKS) anastomosis was added to the proximal ascending aorta with a direct end-to-side anastomosis (Figure 1, $E$ ). In patients 6 to 9 , the descending aorta was anastomosed to the aortic arch after a longitudinal incision was made in the lesser curvature of the aortic arch to the ascending aorta. In patients 8 and 9, a longitudinal incision was made in the anterior wall of the descending aorta. The proximal main pulmonary artery was anastomosed to this confluence in patients 6 to 9 (Figure $1, F$ and $G$ ).

\section{Computational Simulation Methods}

Geometries and meshes. The details of our computational analysis methods have been described. ${ }^{13}$ Postoperative data were acquired by thinslice, early-phase enhanced multidetector-row computed tomography (CT). Image data in a Digital Imaging and Communications in Medicine format were transferred into 3-dimensional patient-specific geometries using the medical imaging software Real INTAGE (KGT Inc, Tokyo, Japan). All constructed geometric sizes were evaluated with accurate measurement from the original Digital Imaging and Communications in Medicine files. All patient geometries are shown in Figure 1.

Computational meshes were created with the commercial software ANSYS-ICEM 12.0 (ANSYS Inc, Tokyo, Japan). More than 1,000,000 cells with tetrahedral meshes and 5 boundary-fitted prism layers were generated. The mesh numbers were defined from our previous mesh refinement study, ${ }^{13}$ and the prism layers were generated at the boundaries to measure accurate WSS at near wall regions.

\section{Boundary Conditions}

To imitate the flow around the valve leaflets, inlet boundaries for the pulmonary artery and the ascending aorta were extended 20 times their diameters to develop velocity profiles and give mass-flow corrected by the BSA of each patient at the time the CT scans were performed. Cardiac outputs were set at $4.3 \mathrm{~L} / \mathrm{min} / \mathrm{m}^{2}$. Echocardiography showed pulsatile flow profiles through the pulmonary and aortic valves.

The outlet boundaries for the arch branches, descending aorta, and bilateral coronary arteries were extended 60 to 70 times of each vessel diameter, and zero pressure gradient conditions were set to obtain sufficient pressure recoveries and to imitate reflex waves from the peripheral portion. ${ }^{13}$ The vessel walls, including the extended boundary walls, were assumed to be rigid.

\section{Turbulent Pulsatile Flow Simulations}

The finite volume solver package ANSYS-FLUENT 12.0 (ANSYS Inc) was used to solve the Navier-Stokes equation of incompressible transient Newtonian fluid. The blood properties were set as follows: density $\rho=1060 \mathrm{~kg} / \mathrm{m}^{3}$ and viscosity $\mu=4.0 \times 10^{-3} \mathrm{~kg} / \mathrm{m} / \mathrm{s}$. Because the Reynolds number was approximately 4000 in the peak systolic phase, we analyzed turbulent flow simulation using the standard k- $\varepsilon$ models. ${ }^{6}$

In the transient flow simulation, each time step was set to $10^{-5}$ seconds to reduce the Courant number to the sufficient level. The convergence criteria were set to $10^{-5}$ times the residual for all the degrees of the parameters at each time step.

\section{Energy Loss and Wall Shear Stress}

From the calculated results, we evaluated EL and WSS.

Total pressure (TP) was defined by the following formula:

$$
T P=\frac{1}{2} \rho|\vec{u}|^{2}+P
$$

where $\vec{u}$ and $\mathrm{P}$ indicate velocity and pressure, respectively. EL was defined with the loss of TP and total cardiac flow.

$$
E L=\sum_{\text {Inlets }}\left(\frac{1}{2} \rho|\vec{u}|^{2}+P\right) Q-\sum_{\text {outles }}\left(\frac{1}{2} \rho|\vec{u}|^{2}+P\right) Q
$$

where Q indicates the blood flow amount. The inlets indicate the pulmonary artery and the ascending aorta in the patients with AS, and the pulmonary 


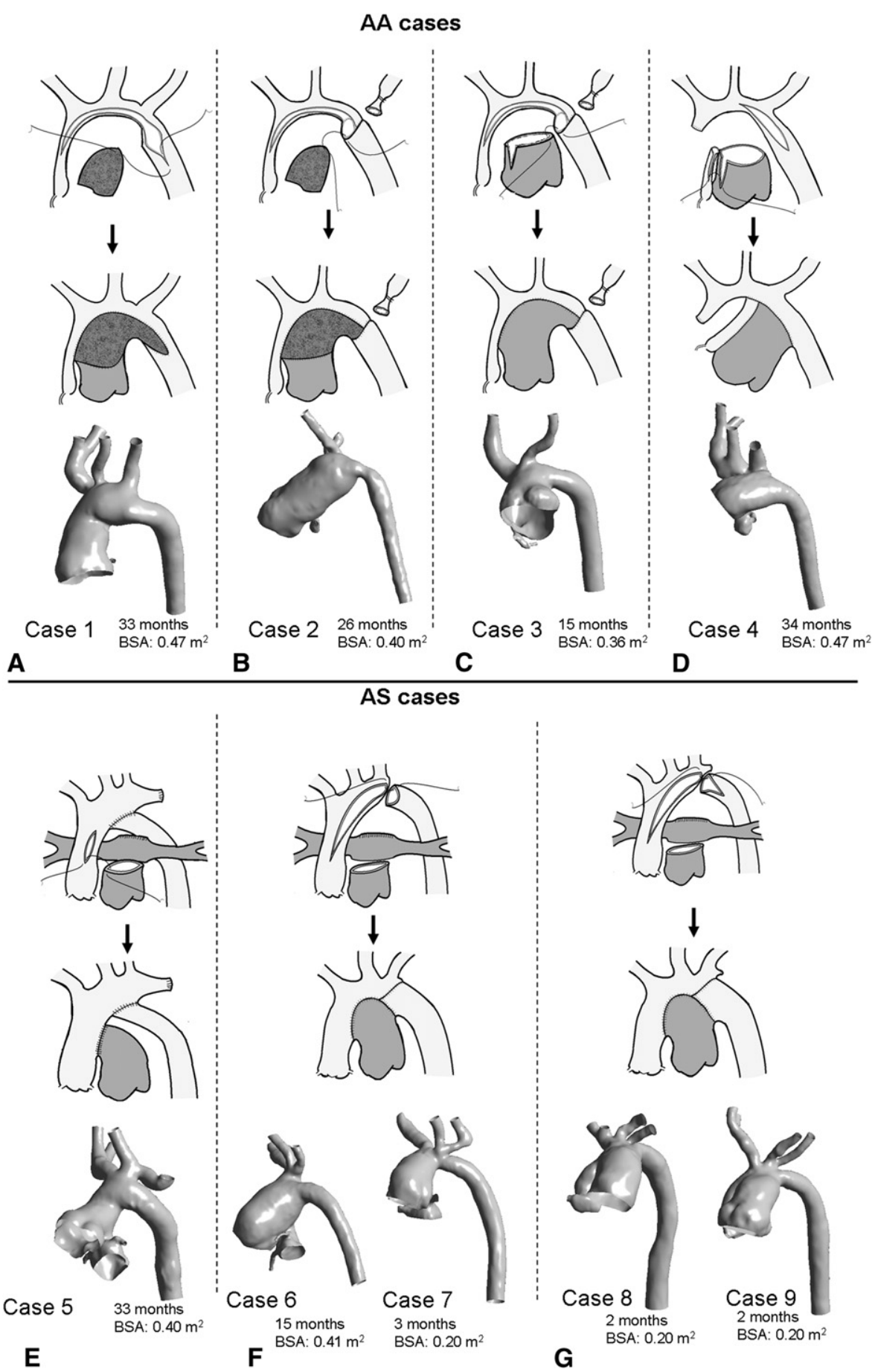

FIGURE 1. Surgical procedure and postoperative 3-dimensional geometry of each patient. A-D, Patients with AA. E-H, Patients with AS. A, Patient 1 underwent arch reconstruction with a homograft patch after a longitudinal incision was made in the lesser curvature of the aortic arch. B, Patient 2 underwent arch reconstruction with a homograft patch after the isthmus, and the periductal tissue was completely excised. C, Patient 3 underwent arch reconstruction without patch material. The main pulmonary artery was anastomosed to the ascending aorta, aortic arch, and descending aorta after the isthmus and periductal tissue were completely excised. D, Patient 4 underwent arch reconstruction without patch material. The ascending aorta was transected and anastomosed in a side-to-side fashion to the main pulmonary artery truncating the main pulmonary artery, and then was anastomosed to the distal aortic arch in an end-to-side fashion. E, Patient 5 underwent arch repair and DKS anastomosis separately. F, Patients 6 and 7 underwent the same procedure. The main pulmonary artery, descending aorta, and aortic arch were reconstructed together in a single anastomosis after a sufficient incision was made to the ascending aorta. G, Patients 8 and 9 underwent a modified procedure as in E. Before the arch reconstruction, a longitudinal incision was made in the anterior wall of the descending aorta. $A A$, Aortic atresia; $B S A$, body surface area; $A S$, aortic stenosis. 


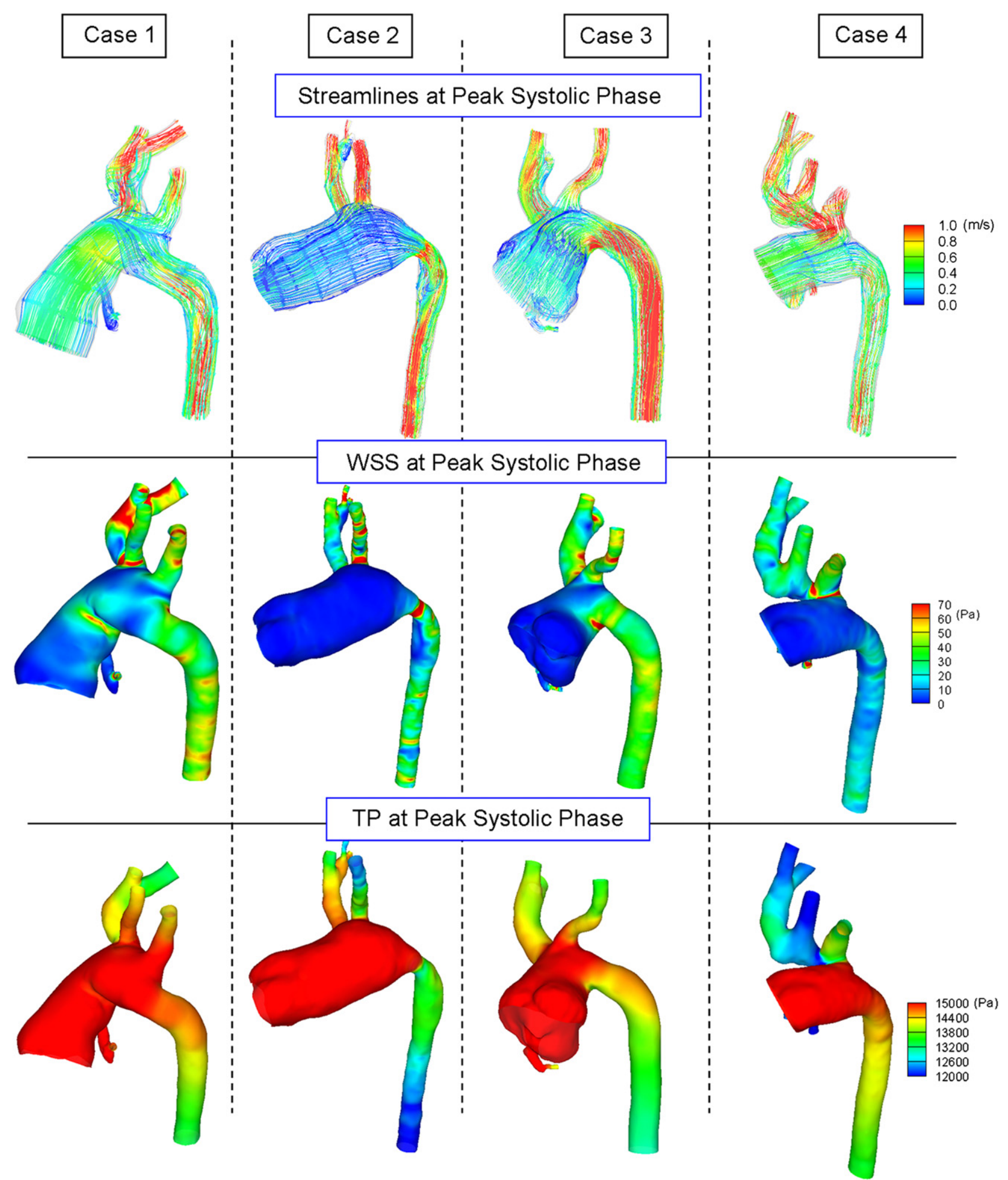

FIGURE 2. Streamlines, wall shear stress (WSS), and total pressure (TP) distribution of patients with AA.

artery in the patients with AA, and the outlets indicate the descending aorta, arch branch vessels, and coronary arteries. WSS was also calculated in all models.

\section{RESULTS}

The streamlines and contour plots of the WSS and TP at peak systolic times are shown in Figures 2 and 3. EL profiles 


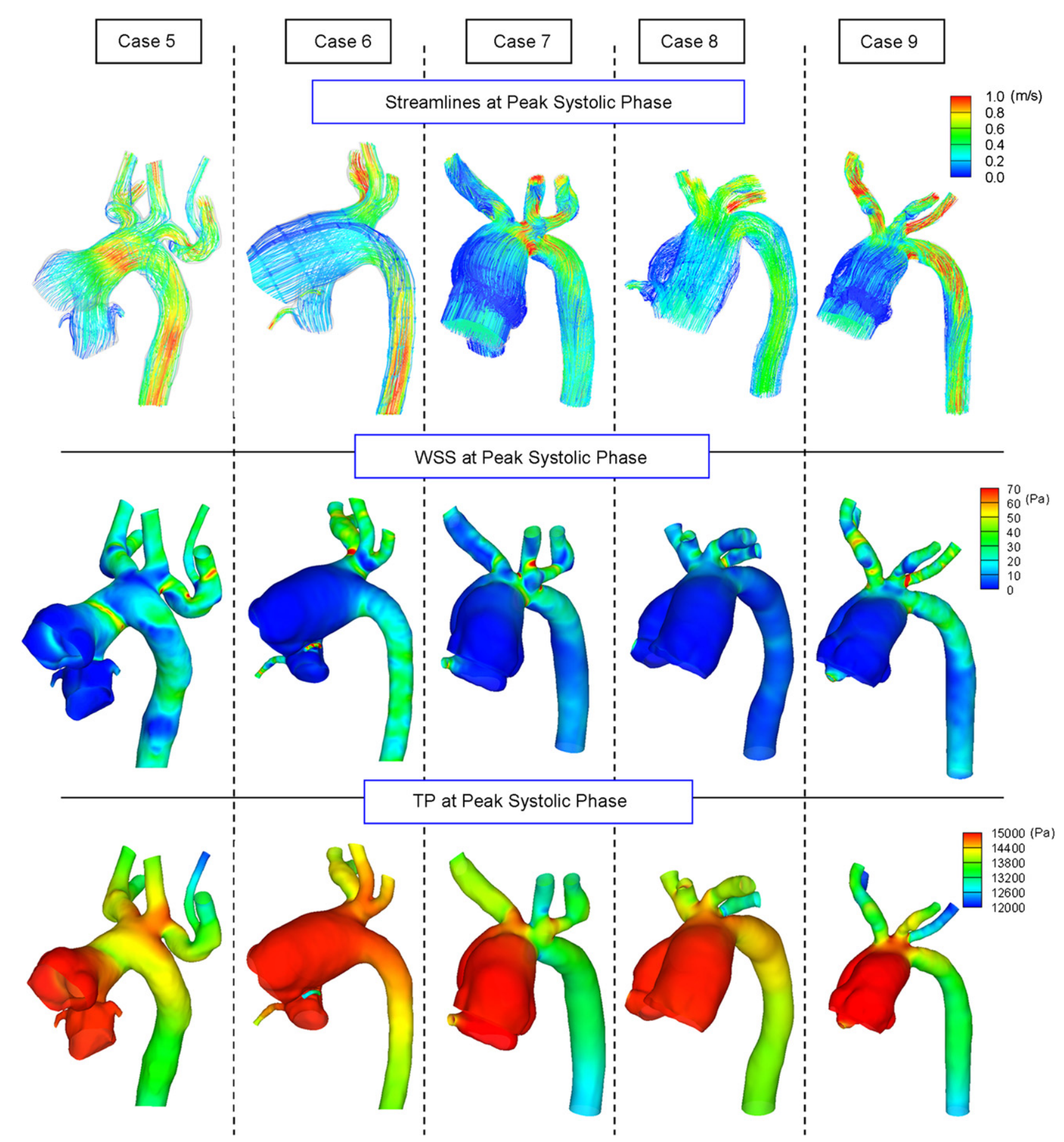

FIGURE 3. Streamlines, wall shear stress (WSS), and total pressure (TP) distribution of patients with AS.

during 1 cardiac cycle are shown in Figure 4. We evaluated the WSS and TP at the peak systolic times because the EL in all cases was highest at these times. ${ }^{13}$

\section{Patients With Aortic Atresia}

Reconstructed arch angles were affected by the procedures (Figures 1 and 2), and 8 patients had no stenosis in the distal anastomosis site. Patient 2 had slight recoarctation.
Flow acceleration was observed in the sharply curved arch in patients 2 and 3, and the arch branches in patient 4 . WSS increase was observed in the regions where the flow accelerations started and TP decreased at these sites.

Compared with patient 2, patient 1 had a large arch space and smoothly curved reconstructed arch with no flow acceleration. The WSS was maintained at a low level, although it increased slightly in a patch-kinking portion, and EL was 
Case 1

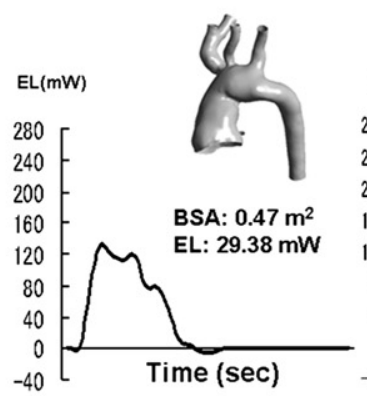

Case 2

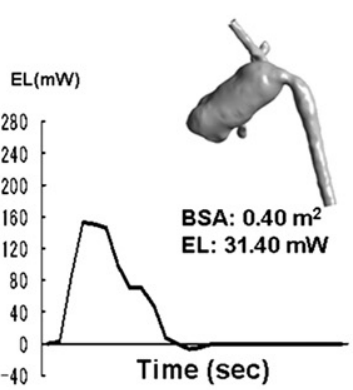

Case 3

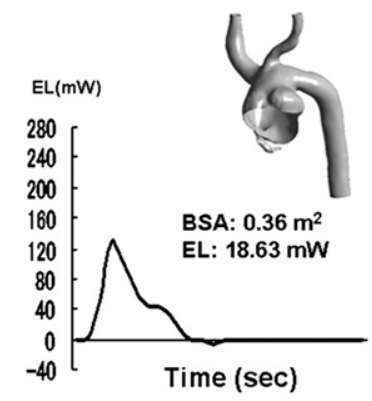

Case 4

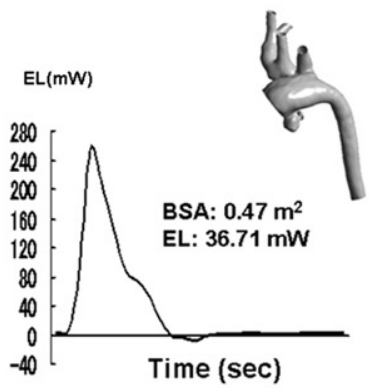

Case 5

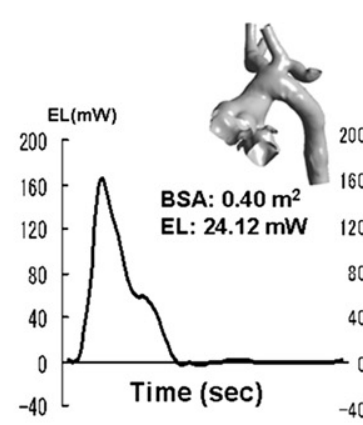

Case 6

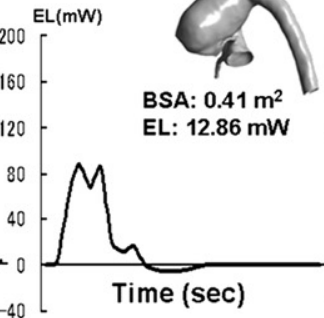

Case 7

Case 8

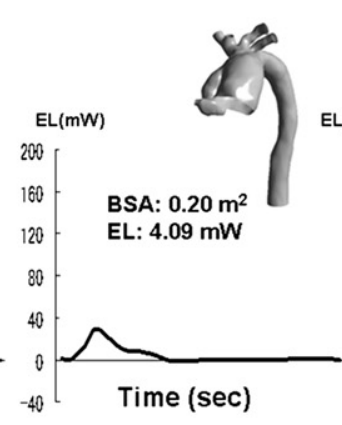

Case 9

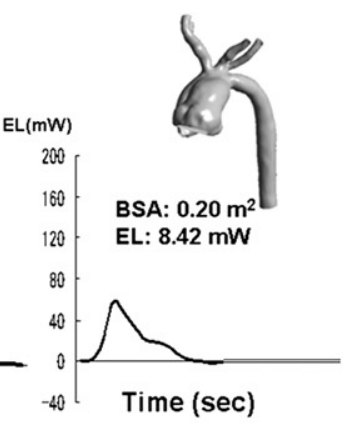

\section{A}
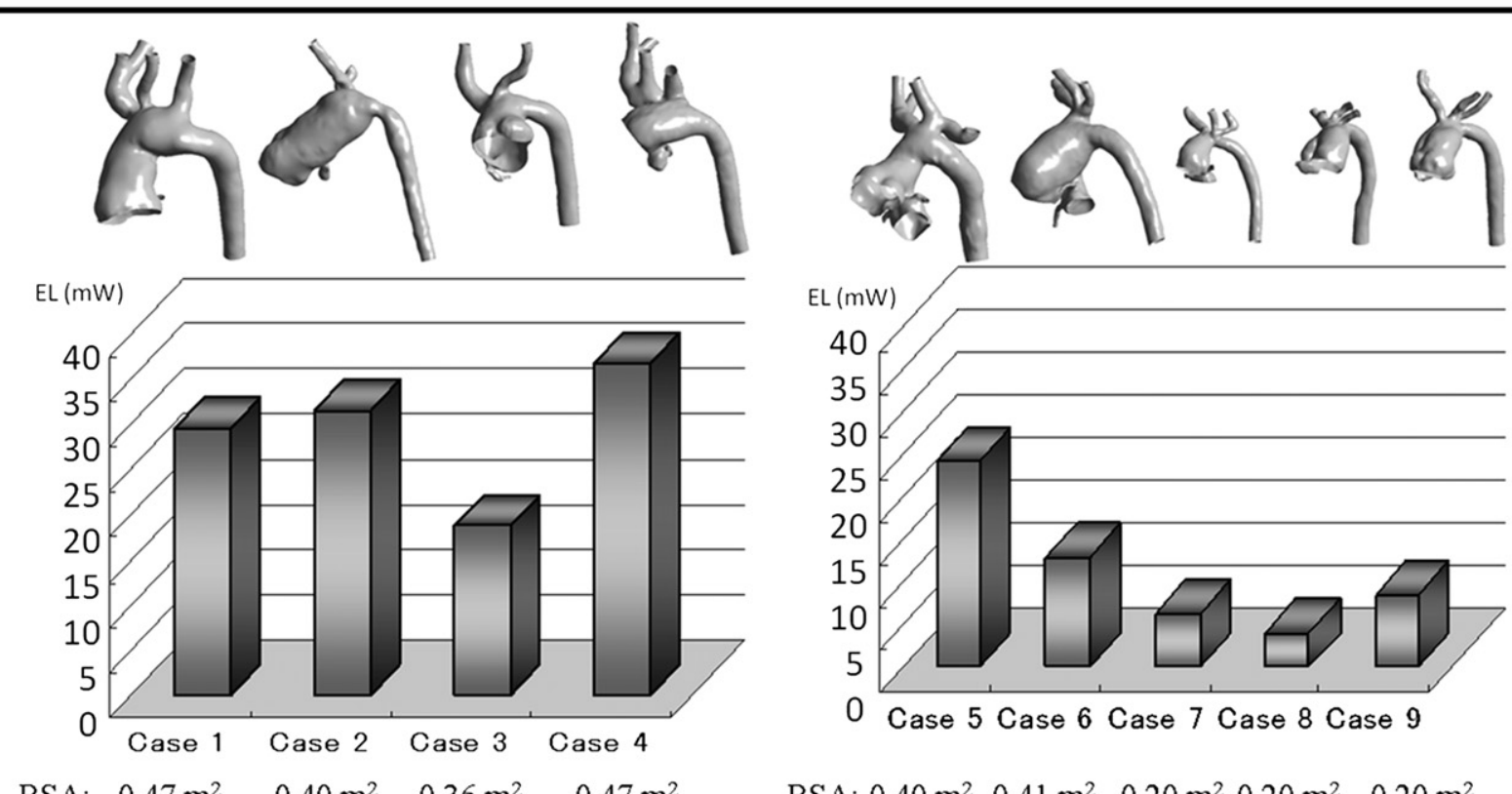

BSA: $\quad 0.47 \mathrm{~m}^{2} \quad 0.40 \mathrm{~m}^{2} \quad 0.36 \mathrm{~m}^{2} \quad 0.47 \mathrm{~m}^{2}$

BSA: $0.40 \mathrm{~m}^{2} \quad 0.41 \mathrm{~m}^{2} \quad 0.20 \mathrm{~m}^{2} \quad 0.20 \mathrm{~m}^{2} \quad 0.20 \mathrm{~m}^{2}$

\section{B}

FIGURE 4. A, Energy loss (EL) profiles during 1 cardiac cycle of all patients. B, Mean EL values during 1 cardiac cycle of all patients. BSA, Body surface area.

low. The EL profile during 1 cardiac cycle had more than 1 peak during the peak systolic phase (Figure 4). A relatively small turbulent flow in the patch-kinking portion disturbed the flow and generated several peaks, but overall it had sufficiently low EL for the patient's body size. Patient 2 had a large main pulmonary artery portion supplemented with 

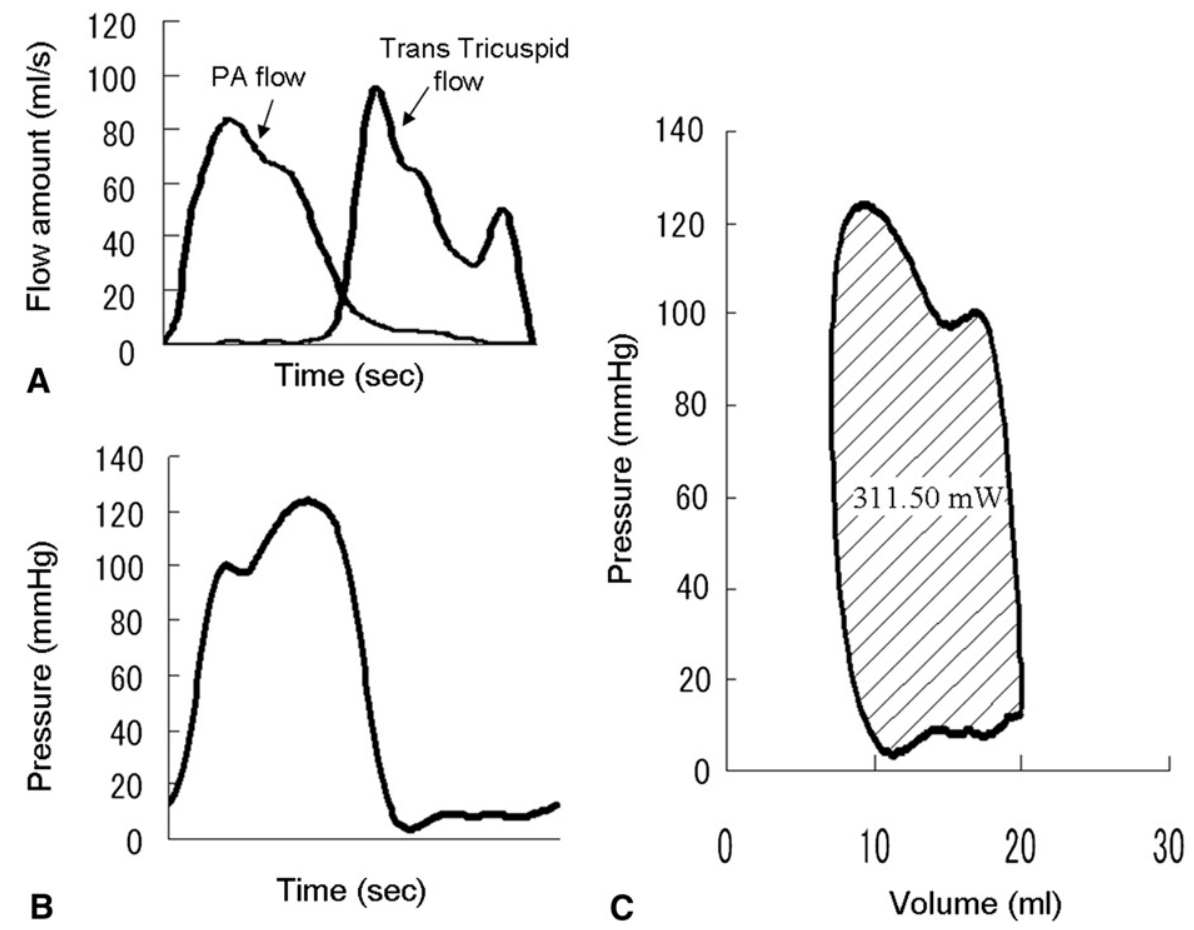

FIGURE 5. Provisory pressure-volume loop of a patient with hypoplastic left heart syndrome. A, Flow amount of pulmonary valve and atrioventricular valve assuming $4.3 \mathrm{~L} / \mathrm{min} / \mathrm{m}^{2}$. B, Ventricular pressure of the systemic right ventricle. C, Hypothetic pressure-volume loop. PA, Pulmonary artery.

the homograft patch, but a space that was slightly too wide in the main pulmonary artery caused a sharp arch angle in the reconstructed arch. The reconstructed arch also had a slight recoarctation, and a $5 \mathrm{~mm} \mathrm{Hg}$ pressure decrease was observed on catheter examination; thus, maximum $1.7 \mathrm{~m} / \mathrm{s}$ flow acceleration was simulated. Although the stenosis was not clinically significant, but rather mild, the sharp arch angle and diameter change at the anastomosis site resulted in relatively high WWS and high EL.

Patients 3 and 4 underwent arch reconstruction with no exogenous patch material. Patient 3 had sufficient anastomosis space and almost no pressure decrease in the distal aortic arch, but the arch angle was acute. Flow was accelerated toward the descending aorta (maximum velocity, $1.1 \mathrm{~m} / \mathrm{s}$ ), and the local WSS increase was detected proximal to the distal anastomosis site. The TP decrease was detected at the same portion. Patient 4 had a smooth aortic arch angle, where only a small amount of WSS and EL were observed in the lesser curvature site of the aortic arch. However, the trunk created with the main pulmonary artery and ascending aorta was anastomosed too distal of the aortic arch. The neck vessels originated at only 1 orifice, where a high flux flow supplying the whole upper half of the body passed, resulting in increased EL.

\section{Patients With Aortic Stenosis}

Flow collisions from the ascending aorta and main pulmonary artery affected the flow acceleration and EL results.
Flow collision was avoided by creating a large anastomosis space and relaxing flow acceleration. Reconstructed arch angles affected both the WSS increase and the TP decrease just as in the patients with AA.

In patient 5, flow acceleration due to flow collisions was observed in the DKS anastomosis site, where the WSS increased and the TP decreased prominently. Moreover, the reconstructed arch had a sharp angle proximal to but past the DKS anastomosis site. In patient 6, the anastomosis site had a relatively large space, and the reconstructed arch had a sufficiently smooth angle. There were no flow accelerations or WSS increases observed, and sufficient energy was preserved. Patient 7 underwent the same procedure as in patient 6 and had low WSS and EL. In patient 8 , we created a smoother arch angle and larger anastomosis space than in patient 7 , which preserved more energy. Although patient 9 underwent the same procedure as patient 8, the TP decreased and EL increased more than in patient 8 , but these were trivial and within sufficiently small levels.

The EL profile during 1 cardiac cycle (Figure 4) and comparison of EL among patients showed that those with large body size (BSA) expended more EL and that those with multiple small EL peaks during 1 cardiac cycle (eg, patients 1 and 6) had a larger anastomosis space and less EL. These multiple peaks were caused by secondary flow in a wide anastomosis space where slightly disturbed flow changed the distributions to the outlets several times during systolic phase. Patients 7 to 9 had a small body 
size, and EL decreased. Patients with AA had no flow collisions, but it was difficult to create a smooth arch angle and they had greater EL than the patients with AS (Figure 5).

\section{DISCUSSION}

The interest in cardiac function of a single right ventricle after the Norwood procedure has been increasing, because the postoperative reconstructed arch can increase the afterload for several reasons, including decreased vessel elastic properties ${ }^{12}$ distal arch stenosis caused by the recoarctation, ${ }^{11}$ or turbulence inside the complicated anastomosis site. ${ }^{13}$ Because of the difficulty in measuring clinically energetic efficiencies in the reconstructed arch and in detecting factors that worsen the cardiac workload in actual patients, detailed flow was visualized using the patientspecific CFD. Single ventricular workload causing longterm ventricular deterioration and vascular stress causing long-term vascular stiffness were estimated with EL and WSS, respectively. In both the AA and AS cases, a large TP decrease occurred almost at the same place where WSS markedly increased, such as in the sharply angled reconstructed arch. WSS caused by the turbulent flow shear was proven to increase EL.

Previous patient-specific CFD models of congenital heart surgery mainly focused on flow distribution and EL in total cavopulmonary connection (TCPC). ${ }^{14-19}$ However, if we take the definition of EL in equations (1) and (2) into account, TCPC is a low-pressure and slow-velocity system expected to produce relatively low TP and low EL, compared with an arterial system that is a high-pressure and fast-velocity system expected to generate greater EL. Our calculated results showed greater EL than results from previous TCPC studies. ${ }^{16-19}$ If the single ventricular physiology is considered to be like a serial circuit, and EL is considered as a part of the energy produced from ventricular ejection power, EL influences the cardiac afterload and is expected to be greater in the reconstructed arch than in TCPC.

To assess the EL for the ventricular workload, we estimated ventricular ejection power by calculating the internal area of a provisory pressure-volume loop of a patient with hypoplastic left heart syndrome (Figure 5). Transatrioventricular valve (tricuspid valve) flow was demonstrated by echocardiography corrected with BSA, just like inlet boundary conditions, and the ventricular volume change was calculated. The intraventricular pressure curves obtained with the catheter examination were superposed with the electrocardiogram. Although the end-diastolic volume was unknown, the internal area, which indicated the flow ejection energy, was calculated. Produced energy totaled $311.50 \mathrm{~mW}$ in a patient with a BSA of $0.40 \mathrm{~m}^{2}$. Although this method is only an approximate one, we conjectured how large the calculated ELs were compared with the cardiac work itself and found they were approximately
$3 \%$ to $15 \%$ of the generated energy from the systemic ventricle and considered to be non-negligible.

Results from the patients with AA suggested that the smooth angle reducing WSS could be accomplished by the longitudinal incision of the descending aorta, as in patients 1 and 4. Burkhart and colleagues ${ }^{8}$ introduced the "interdigitating technique," in which the descending aorta is incised longitudinally. This method prevents recoarctation and maintains a smooth arch angle with the longitudinal incision in the descending aorta. Results from the patients with AS suggest that a combination of arch repair and DKS anastomosis in a single anastomotic space, first introduced by Ishino and colleagues, ${ }^{7}$ ensures sufficient anastomotic space, which prevents flow collisions from the ascending aorta and pulmonary artery, and maintains a smooth arch angle with trivial WSS increase and preserved energy.

\section{Study Limitations}

With computational models, there are usually difficulties in simulating some biological changes. Degeneration, cicatrization, or calcification, which are all known to be essential problems, especially for patch materials, ${ }^{7}$ could not be included in our model. We also did not create vessel elasticity or compliance. In addition to the numeric solver of fluid and structure interaction, exact material property information of the postoperative vessel wall is needed. Because of the dense adhesion around the mobilized and sutured vessel wall, rigid wall approximation is justified to some extent. Because of the technical limitations and lack of knowledge of the postoperative vessel properties, we evaluated WSS distributions, and the expected high WSS region became more intense.

It is also difficult for computer models to take patient growth into account. Future studies should include larger patient populations, repeat the evaluations of all the patients studied with the same calculation methods, and compare the results to clarify the long-term changes and outcomes.

\section{CONCLUSIONS}

With the use of CFD techniques, we analyzed the hemodynamic details of 9 patients who underwent the Norwood procedure and different types of arch reconstruction methods. In regard to EL and WSS, a large anastomosis space to avoid recoarctation and a smooth arch angle were important for the overall reduction of the cardiac workload. The longitudinal incision of the descending aorta in the patients with AA and the combination of arch repair and DKS in the single anastomosis in the patients with AS were effective in the Norwood procedure.

\section{References}

1. Bacha EA, Larrazabal LA, Pigula FA, et al. Measurement of technical performance in surgery for congenital heart disease: the stage I Norwood procedure. J Thorac Cardiovasc Surg. 2008;136:993-7. 
2. Karamichalis JM, Thiagarajan RR, Liu H, et al. Stage I Norwood: optimal technical performance improves outcomes irrespective of preoperative physiologic status or case complexity. J Thorac Cardiovasc Surg. 2010;139:962-8.

3. Stasik CN, Gelehrter S, Goldberg CS, et al. Current outcomes and risk factors for the Norwood procedure. J Thorac Cardiovasc Surg. 2006;131:412-7. Erratum in: J Thorac Cardiovasc Surg. 2007;133:602.

4. Norwood WI, Kirklin JK, Sanders SP. Hypoplastic left heart syndrome: experience with palliative surgery. Am J Cardiol. 1980;45:87-91.

5. Jonas RA, Lang P, Hansen D, Hickey P, Castaneda AR. First-stage palliation of hypoplastic left heart syndrome. The importance of coarctation and shunt size. J Thorac Cardiovasc Surg. 1986;92:6-13.

6. Fraser CD Jr, Mee RB. Modified Norwood procedure for hypoplastic left heart syndrome. Ann Thorac Surg. 1995;60(6 Suppl):S546-9.

7. Ishino K, Stumper O, De Giovanni JJ, et al. The modified Norwood procedure for hypoplastic left heart syndrome: early to intermediate results of 120 patients with particular reference to aortic arch repair. J Thorac Cardiovasc Surg. 1999;117: 920-30.

8. Burkhart HM, Ashburn DA, Konstantinov IE, et al. Interdigitating arch reconstruction eliminates recurrent coarctation after the Norwood procedure. J Thorac Cardiovasc Surg. 2005;130:61-5.

9. Griselli M, McGuirk SP, Stumper O, et al. Influence of surgical strategies on outcome after the Norwood procedure. J Thorac Cardiovasc Surg. 2006;131: 418-26.

10. Bautista-Hernandex V, Marx GR, Gauvreau K, et al. Coarctectomy reduces neoaortic arch obstruction in hypoplastic left heart syndrome. J Thorac Cardiovasc Surg. 2007;133:1540-6.

11. Larrazabal LA, Selamet Tierney ES, Brown DW, et al. Ventricular function deteriorates with recurrent coarctation in hypoplastic left heart syndrome. Ann Thorac Surg. 2008;86:869-74.

12. Cardis BM, Fyfe DA, Mahle WT. Elastic properties of the reconstructed aorta in hypoplastic left heart syndrome. Ann Thorac Surg. 2006;81:988-91.
13. Qian Y, Liu JL, Itatani K, Miyaji K, Umezu M. Computational hemodynamic analysis in congenital heart disease: simulation of the Norwood procedure. Ann Biomed Eng. 2010;37:2302-13.

14. Pekkan K, de Zelicourt D, Ge L, et al. Physics-driven CFD modeling of complex anatomical cardiovascular flows-a TCPC case study. Ann Biomed Eng. 2005;33: 284-300.

15. de Zelicourt DA, Pekkan K, Parks J, et al. Flow study of an extracardiac connection with persistent left superior vena cava. J Thorac Cardiovasc Surg. 2006;131:785-91.

16. Whitehead KK, Pekkan K, Kitajima HD, et al. Nonlinear power loss during exercise in single-ventricle patients after the Fontan: insights from computational fluid dynamics. Circulation. 2007;116(11 Suppl):I165-71.

17. Wang C, Pekkan K, de Zelicourt D, et al. Progress in the CFD modeling of flow instabilities in anatomical total cavopulmonary connections. Ann Biomed Eng. 2007;35:1840-56.

18. Marsden AL, Vignon-Clementel IE, Chan FP, Feinstein JA, Taylor CA. Effects of exercise and respiration on hemodynamic efficiency in CFD simulations of the total cavopulmonary connection. Ann Biomed Eng. 2007;35:250-63.

19. Itatani K, Miyaji K, Tomoyasu T, et al. Optimal conduit size of the extracardiac Fontan operation based on energy loss and flow stagnation. Ann Thorac Surg. 2009;88:565-72.

20. Migliavacca F, Yates R, Pennati G, et al. Calculating blood flow from Doppler measurements in the systemic-to-pulmonary artery shunt after the Norwood operation: a method based on computational fluid dynamics. Ultrasound Med Biol. 2000;26:209-19.

21. Bove EL, Migliavacca F, de Leval MR, et al. Use of mathematic modeling to compare and predict hemodynamic effects of the modified Blalock-Taussig and right ventricle-pulmonary artery shunts for hypoplastic left heart syndrome. J Thorac Cardiovasc Surg. 2008;136:312-20.

22. Hsia TY, Migliavacca F, Pennati G, et al. Management of a stenotic right ventricle-pulmonary artery shunt early after the Norwood procedure. Ann Thorac Surg. 2009;88:30-8. 